\title{
Direct Measurement of Dirac Point Energy at the Graphene/Oxide Interface
}

\author{
Kun Xu, ${ }^{*, t, \neq \perp}$ Caifu Zeng, ${ }^{\S, \perp}$ Qin Zhang, ${ }^{\dagger, \|}$ Rusen Yan, ${ }^{\dagger, \|}$ Peide Ye, ${ }^{\ddagger}$ Kang Wang, ${ }^{\S}$ Alan C. Seabaugh,, \\ Huili Grace Xing," John S. Suehle, ${ }^{\dagger}$ Curt A. Richter, ${ }^{\dagger}$ David J. Gundlach, ${ }^{\dagger}$ and N. V. Nguyen*, ${ }^{\dagger}$ \\ ${ }^{\dagger}$ Semiconductor and Dimensional Metrology Division, National Institute of Standards and Technology, Gaithersburg, Maryland \\ 20899, United States \\ "Purdue University, West Lafayette, Indiana 47907, United States \\ ${ }^{\S}$ University of California-Los Angeles, Los Angeles, California 90095, United States \\ "University of Notre Dame, Notre Dame, Indiana 46556, United States
}

ABSTRACT: We report the direct measurement of the Dirac point, the Fermi level, and the work function of graphene by performing internal photoemission measurements on a graphene $/ \mathrm{SiO}_{2} / \mathrm{Si}$ structure with a unique optical-cavity enhanced test structure. A complete electronic band alignment at the graphene $/ \mathrm{SiO}_{2} / \mathrm{Si}$ interfaces is accurately established. The observation of enhanced photoemission from a one-atom thick graphene layer was possible by taking advantage of the constructive optical interference in the $\mathrm{SiO}_{2}$ cavity. The photoemission yield was found to follow the well-known linear density-of-states dispersion in the vicinity of the Dirac point.
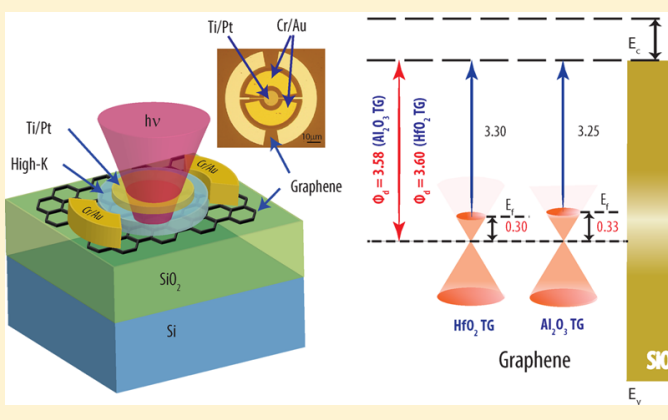
At the flat band condition, the Fermi level was extracted and found to reside $3.3 \mathrm{eV} \pm 0.05 \mathrm{eV}$ below the bottom of the $\mathrm{SiO}_{2}$ conduction band. When combined with the shift of the Fermi level from the Dirac point, we are able to ascertain the position of the Dirac point at $3.6 \mathrm{eV} \pm 0.05 \mathrm{eV}$ with respect to the bottom of the $\mathrm{SiO}_{2}$ conduction band edge, yielding a work function of $4.5 \mathrm{eV} \pm 0.05 \mathrm{eV}$ which is in an excellent agreement with theory. The accurate determination of the work function of graphene is of significant importance to the engineering of graphene-based devices, and the measurement technique we have advanced in this Letter will have significant impact on numerous applications for emerging graphene-like 2-dimensional material systems.

KEYWORDS: Graphene, work function, internal photoemission, band alignment, graphene-insulator-semiconductor

ince the pioneering work of Novoselov et al. in 2004, ${ }^{1}$ $\checkmark$ graphene has attracted an immense amount of interest from many related disciplines. ${ }^{2,3}$ Fundamental knowledge of the physical properties of graphene and the physical mechanisms governing the electrical operation of graphenebased devices has grown dramatically. ${ }^{4}$ With the recent success of large area chemical vapor deposition (CVD) growth of graphene, ${ }^{5}$ industrial applications such as transparent electrodes, ${ }^{6}$ field-effect transistors (FETs), ${ }^{7}$ and quantum well devices ${ }^{8}$ are becoming more promising. Many studies have been conducted to characterize the various physical properties of graphene, including the work function, which is one of the most important electronic parameters. Among the numerous investigations by techniques such as Kelvin probe measurements, ${ }^{9-11}$ ab initio calculations, ${ }^{9,12}$ and recently by capacitance-voltage measurements, ${ }^{13}$ the values of work function scatter in a rather wide range from 4.2 to $5.0 \mathrm{eV}$. Experimentally, the work function may be found to vary depending on the type of metal contact due to interactions between the graphene and the metal, which may result in pinning of the work function. ${ }^{9-13}$ Surprisingly, there is little information on the intrinsic electronic band alignment of the graphene/oxide interface to date, despite its important role in the design, fabrication, and characterization of graphene-based devices. For example, the accurate band alignment between graphene and another material determines how effectively to turn on and off a device, such as a graphene FET, with an applied electric field. The band alignment between condensed phases is undoubtedly affected by the electronic and chemical nature of the interface and the interaction between the two materials. Thus, the direct measurement of the intrinsic band alignment of graphene allows one not only to understand the electronic properties of the interface but also to potentially succeed in designing and implementing advanced graphene devices.

Internal photoemission (IPE) spectroscopy has been shown to be a robust technique to determine electronic band alignment in heterostructure systems. ${ }^{14-16}$ By inducing the

Received: October 2, 2012

Revised: December 3, 2012 
transition of carriers from one material to another through optical excitation, IPE can directly measure the band offsets at the interfaces between graphene and oxides. However, as a 2-D material with a single atomic layer thickness, graphene poses many experimental challenges that need to be resolved if we are to conduct effective IPE spectroscopy. These include: (1) the actual realization of IPE measurements on the graphene/oxide interface when the nature of the single atomic thickness of graphene inevitably imposes a limit on the photoexcited carrier concentration and (2) the application of existing photoelectric emission models to this 2-D material system. We will discuss these issues throughout the manuscript and demonstrate a unique test structure design that addresses the measurement challenges and permits the characterization of the electronic interfacial properties of this important graphene/oxide system.

Efforts to measure the band alignment between graphene and $\mathrm{SiO}_{2}$ have been made by Yan et al. ${ }^{17}$ using IPE spectroscopy, but due to the optical transparency and the very limited photoexcited carrier concentration in a single atomic layer of graphene, no photoemission from graphene was observed given the detection limit of their measurement. ${ }^{18}$ To overcome this challenge, we employ a $\mathrm{SiO}_{2}$ layer of special design instead of the interference-free thin oxide that is used in conventional IPE measurements and take advantage of the interference-induced absorption peaks that enhance the photoemission signal of electrons from graphene. By using this approach, we are able to experimentally establish a complete and accurate electronic band alignment of the graphene $/ \mathrm{SiO}_{2} / \mathrm{Si}$ system and thus derive the intrinsic work function of graphene.

Figure 1 shows the test structure designed and fabricated for this study. The graphene was grown on copper foil by using a

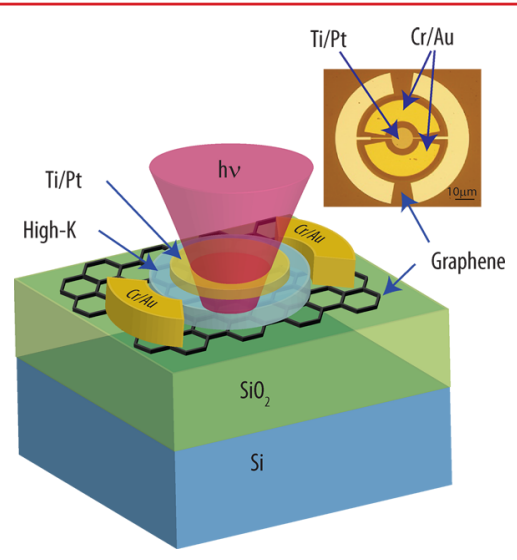

Figure 1. Schematic of the test structure with high- $K /$ graphene $/ \mathrm{SiO}_{2} /$ Si stack. $\mathrm{Al}_{2} \mathrm{O}_{3}$ and $\mathrm{HfO}_{2}$ are used at high- $k$ top gate. The top right picture shows the top view of the device under the optical microscope.

chemical vapor deposition (CVD) method and transferred onto a $n^{++}$doped Si substrate $\left(n_{\mathrm{d}}=10^{19}-10^{20} \mathrm{~cm}^{-3}\right)$ with $300-\mathrm{nm}$ thick thermally grown $\mathrm{SiO}_{2}$ by using a poly(methyl methacrylate) (PMMA) wet transfer method. The PMMA layer was removed with an acetone bath followed by a $\mathrm{H}_{2} / \mathrm{Ar}$ forming gas anneal at $350{ }^{\circ} \mathrm{C}$ for $1 \mathrm{~h}$. A $3 \mathrm{~nm} \mathrm{Al}$ sacrificial layer was deposited on top of the graphene by electron-beam (ebeam) evaporation to prevent the contamination from photoresist residues for better contact and cleaner interface. ${ }^{19}$ A metal layer of $20-\mathrm{nm}-\mathrm{Cr} / 100-\mathrm{nm}-\mathrm{Au}$, used as the contact for graphene, was patterned by photolithography and deposited by e-beam evaporation. A $2 \mathrm{~nm} \mathrm{Al}$ seed layer was deposited by e- beam evaporation and oxidized in atmosphere followed by atomic layer deposition (ALD) of $8 \mathrm{~nm} \mathrm{Al} \mathrm{O}_{3}$ as the top gate dielectric. Structures with $\mathrm{HfO}_{2}$ top gate dielectric were fabricated in a similar fashion with $11.5 \mathrm{~nm}$ of $\mathrm{HfO}_{2}$ grown by ALD after $1 \mathrm{~nm}$ Ti seed layer. A semitransparent 1-nm-Ti/9nm-Pt layer was used for the top gate metal, through which incident photons were able to reach the desired interfaces. The graphene and the high- $k$ oxide outside the active region were etched by reactive-ion-etching (RIE) to isolate each device. A 200-nm-thick Al layer, used for the electrodes for the top gate, was patterned by photolithography and deposited by e-beam evaporation.

The IPE measurement system is comprised of a $150 \mathrm{~W}$ broadband xenon light source in conjunction with a grating monochromator to provide a spectral range from 1.5 to $6 \mathrm{eV}^{20}$ A regulated low noise DC voltage supply provides the bias $\left(V_{\mathrm{G}}\right)$ which is applied to the back gate of the test structure. An electrometer with subpico-Ampere precision records the photo current $(I)$ while the monochromator scans the spectral range. The photoelectric quantum yield $(Y)$ is defined as the ratio of the photocurrent and the flux $(P)$ of the incident light. ${ }^{21}$

Traditionally, the IPE process has been treated as three separate quasi-independent steps: optical generation of electron and/or hole carriers in a solid (emitter), transport of the carriers to the interface, and escape of the carriers over the interface barrier to another solid (collector). Over the years, this approach has been shown to successfully interpret and model IPE experiments in many material interface systems. ${ }^{14}$ Currently, this method has not yet been theoretically investigated as a means to deal with 2-D material systems, generally, and graphene in particular. However, in the following discussion, we will show that this classical model of IPE is still experimentally applicable to the case of graphene for spectral responses near the barrier threshold.

Since graphene is only one atom thick, it is intuitive to consider only the carrier generation and escape steps for carrier transport normal to the 2-D plane of graphene. Powell's model $^{21}$ for the optical excitation in the emitter combined with Fowler's classical electron transmission ${ }^{21,22}$ or escape probability at the emitter/collector interface results in the quantum yield. The yield is expressed as a function of the interface energy barrier height $(\Phi)$ and of the photon energy $(h \nu)$ in the vicinity of $\Phi$ :

$$
Y(h \nu)=A(h \nu)(h \nu-\Phi)^{p}
$$

where $A(h \nu)$ is a factor related to the optical constants of the material and light intensity, and $p$ depends on the shape of the energy distribution function of the excited carriers at the interface of the emitter. The excited carrier distribution is the product of the initial and final energy distributions and the coupling matrix between the initial and final carrier states. Close to the photoemission threshold, the final energy distribution of carriers is considered a weak function of the carrier energy, and it has been shown that the functional form of the excited carrier distribution can be taken to be similar to the initial occupied state distribution. ${ }^{14}$ Due to the unique band structure near the Dirac point, carriers in graphene exhibit the well-known linear distribution $D(E)=|E| / 2 \pi \hbar^{2} \nu_{\mathrm{F}}^{2}$, where $\hbar$ is the reduced Planck constant and $\nu_{\mathrm{F}}$ is the Fermi velocity of graphene. $^{23}$ Therefore we model that the final energy distribution of excited carriers has the same linear shape. This corresponds to the case of $p=3$ where the energy distribution of the excited carriers at the interface has a ramp-like shape in 
the classical model. ${ }^{21}$ The barrier height can be experimentally obtained by plotting $Y^{1 / 3}$ vs $h v$ for graphene/oxide interfaces.

As we have mentioned before, the weak total absorption ${ }^{18}$ in single-layer graphene makes it difficult to obtain an ample amount of excited carriers for measurable and distinguishable IPE signals. Fortunately, the $\mathrm{SiO}_{2}$ sandwiched between graphene and $\mathrm{Si}$ substrate can act as an optical interference cavity that introduces significant modulation to the absorption. Our study shows that the commonly used $300 \mathrm{~nm} \mathrm{SiO} 2$ not only allows the visibility of graphene under a microscope but also provides the necessary modulation that increases the amount of light absorption in graphene for the detection of IPE in the spectral range of interest. To quantitatively analyze the influence of the $\mathrm{SiO}_{2}$ thickness on the photocurrent spectra, we compute the absorptance of light in each layer of our device. This is achieved by building a classical optical model of a multilayer $\mathrm{Ti}-\mathrm{Pt} / \mathrm{Al}_{2} \mathrm{O}_{3} /$ graphene $/ \mathrm{SiO}_{2} / \mathrm{Si}$ structure (as seen in Figure 2a), in which the wavelength-dependent complex refractive indices of each material, measured by spectroscopic ellipsometry, are used. ${ }^{24,25}$ The classical transfer-matrix method described by Heavens ${ }^{26}$ was used in our calculation for
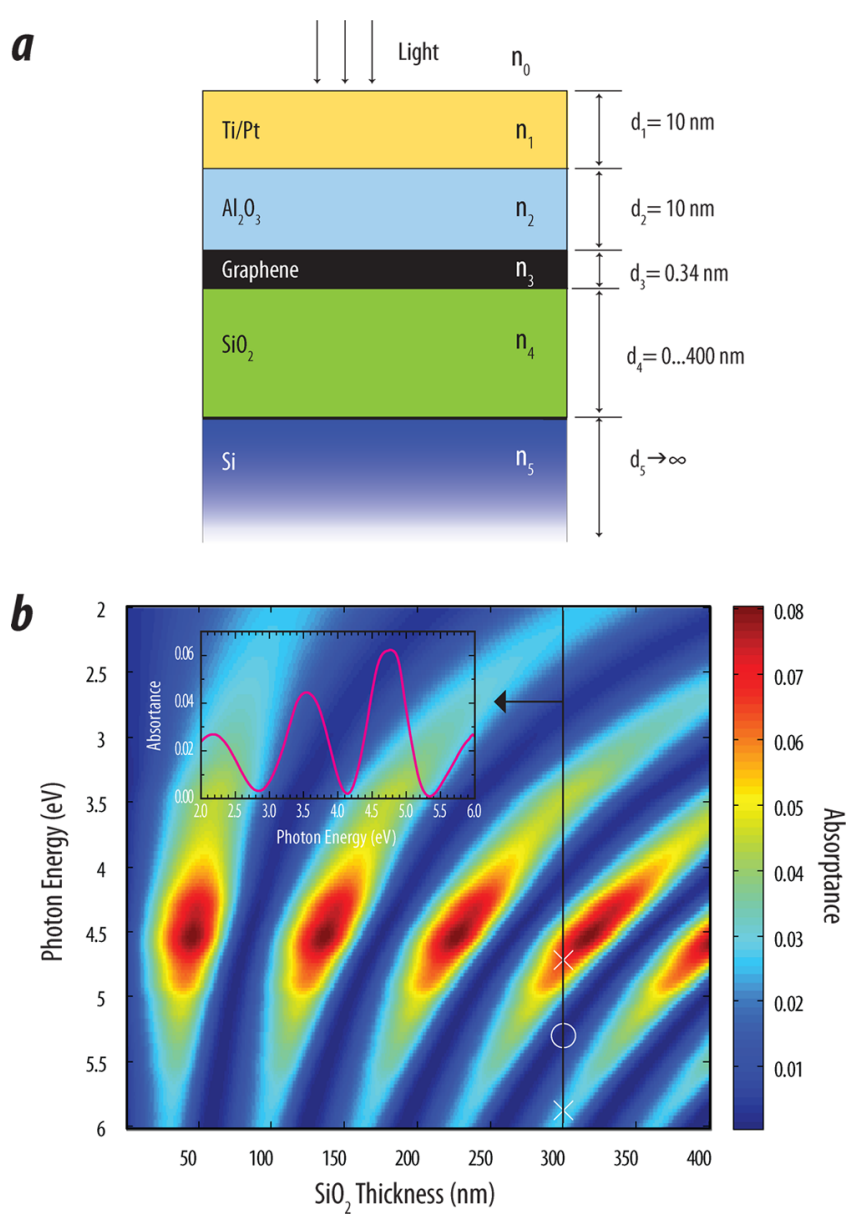

Figure 2. (a) A five-layer model for understanding the effects of oxide thickness on optical absorption in the graphene layer. $n_{0, \ldots, 5}$ 's are the complex refractive indices of air, $\mathrm{Ti} / \mathrm{Pt}, \mathrm{Al}_{2} \mathrm{O}_{3}$, graphene, $\mathrm{SiO}_{2}$, and $\mathrm{Si}$, respectively, while $d_{1} \ldots d_{5}$ are the thickness of each layer. The thickness of $\mathrm{SiO}_{2}$ varies from 0 to $400 \mathrm{~nm}$, and the thickness of $\mathrm{Si}$ is assumed to be infinity. (b) Absorptance spectra in the graphene layer as a function $\mathrm{SiO}_{2}$ thickness. Crosses mark the absorptance peaks, and the circle indicates the minimum at an oxide thickness of $300 \mathrm{~nm}$. The inset plots the absorptance corresponding to the $300 \mathrm{~nm}$ thickness. computing the optical power flow and power absorption at each layer. In Figure $2 b$, the absorptance at the graphene layer is mapped as a function of the $\mathrm{SiO}_{2}$ thickness and the incident photon energy. The regions with warm colors show a maximum of about $10 \times$ higher absorptance than the regions with the lowest absorptance. The constructive interference from $\mathrm{SiO}_{2}$ cavity causes graphene to more efficiently absorb broader light spectra only at certain thicknesses; while in the other regions, absorption in the graphene layer vanishes or falls in the energy range that is not of particular interest to our measurement. The $300 \mathrm{~nm} \mathrm{SiO} 2$ thickness is favorable because absorption peaks dispersed at $3.5 \mathrm{eV}, 4.8 \mathrm{eV}, 5.9 \mathrm{eV}$, and even at $2.2 \mathrm{eV}$ provide the necessary excited carriers in the graphene layer over multiple spectral ranges.

The IPE measurements were performed on samples with 300 $\mathrm{nm} \mathrm{SiO}_{2}$ with a top gate of either $\mathrm{Al}_{2} \mathrm{O}_{3}$ or $\mathrm{HfO}_{2}$. Bias voltages from -20 to $20 \mathrm{~V}$ in steps of $1 \mathrm{~V}$ were applied to the $n^{++}$-doped Si substrate, with graphene grounded and top gate floating. Figure 3 shows the IPE photocurrents for samples with an

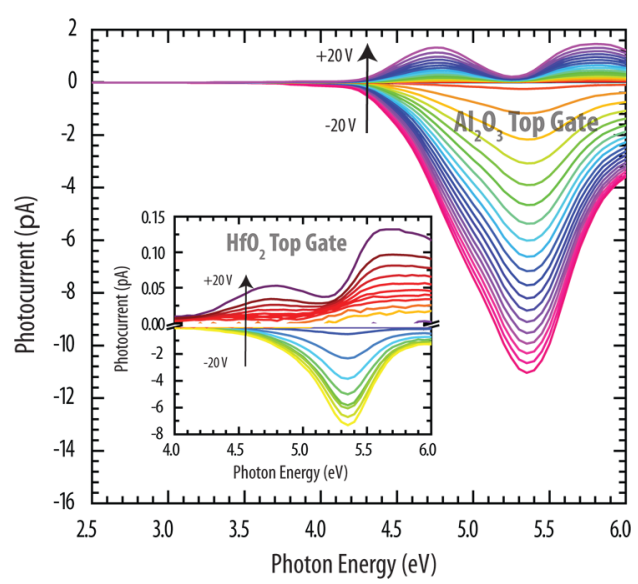

Figure 3. Photoelectric current from graphene to $300 \mathrm{~nm} \mathrm{SiO}{ }_{2}$ with $\mathrm{Al}_{2} \mathrm{O}_{3}$ top gate measured as a function of incident photon energy and with back-gate bias as the parameter. Similar data are shown in the inset for $\mathrm{HfO}_{2}$ top gate. The oscillation is a result of the optical interference in the $\mathrm{SiO}_{2}$ cavity layer.

$\mathrm{Al}_{2} \mathrm{O}_{3}$ top gate and an $\mathrm{HfO}_{2}$ top gate (shown in the inset). The flatband voltage $\left(V_{\mathrm{fb}}\right)$ is determined at the applied voltage when the photocurrent switches direction near the photoemission threshold. ${ }^{16}$ This switch occurs at $0.86 \mathrm{~V}$ for the sample with the $\mathrm{Al}_{2} \mathrm{O}_{3}$ top gate and at $1.9 \mathrm{~V}$ with the $\mathrm{HfO}_{2}$ top gate. Biased above $V_{\mathrm{fb}}$, the photoexcited electrons come from graphene to $\mathrm{SiO}_{2}$. The oscillation observed in the photocurrent spectra is attributed to the optical interference effects related to the thickness of the $\mathrm{SiO}_{2}$. By comparing the photon energy of the maxima in the simulated absorptance spectra with our measured photocurrent, the observed maxima at $\sim 4.8 \mathrm{eV}$ and $\sim 5.8 \mathrm{eV}$ in the photocurrent from graphene $\left(V>V_{\mathrm{fb}}\right)$ in Figure 3 can be attributed to the constructive interference of the absorbance in the sandwiched structure with $300 \mathrm{~nm} \mathrm{SiO}$. Also, at $\sim 5.4 \mathrm{eV}$, we observe minimum photocurrent at the graphene $/ \mathrm{SiO}_{2}$ interface corresponding to the destructive interference, and most light propagates through the oxide and is absorbed in the silicon substrate giving rise to the strong photoemission currents from the $\mathrm{Si} / \mathrm{SiO}_{2}$ interface for both $\mathrm{Al}_{2} \mathrm{O}_{3}$ and $\mathrm{HfO}_{2}$ top gates. This pronounced oscillation produced by optical interference is essential for enhancing the photoelectric yield that enables the observation of distinct 
emission of electrons from the Fermi level of graphene to the conduction band of $\mathrm{SiO}_{2}$.

The barrier height at a given oxide field is determined from the cubic dependence of IPE yield versus photon energy, as plotted in Figure 4. The barrier threshold $\left(\Phi_{\mathrm{e}}\right)$ that appears in
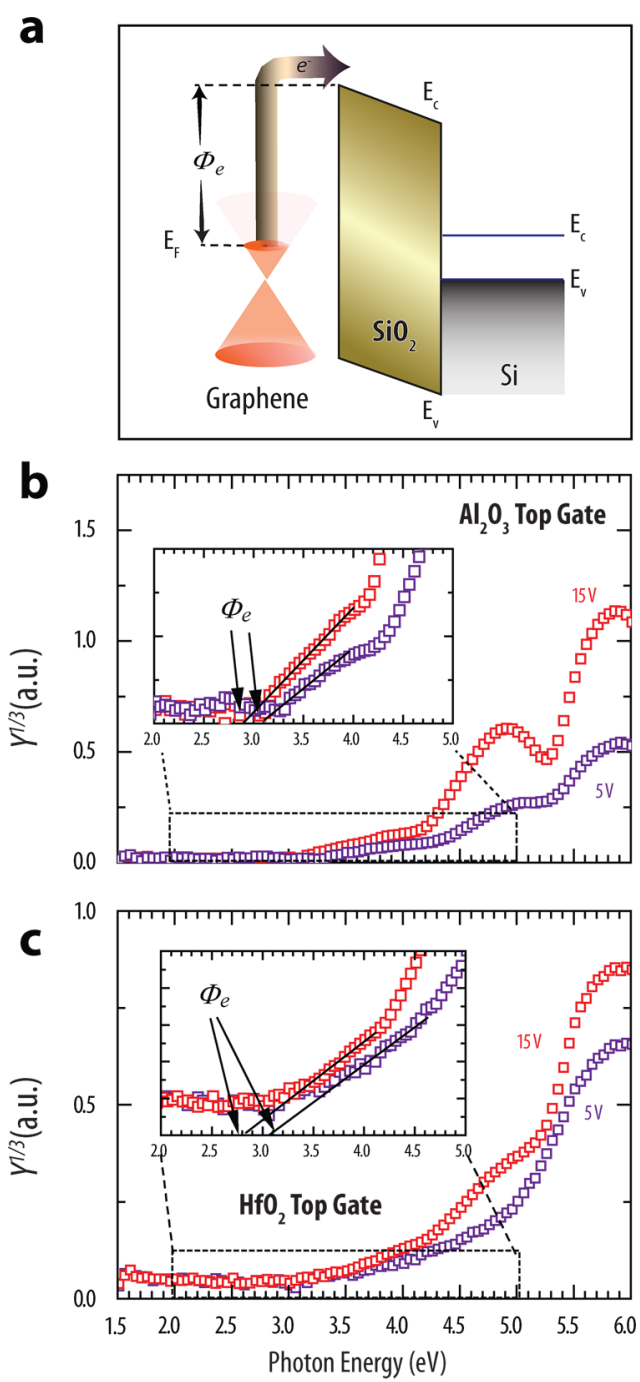

Figure 4. (a) Schematic description of photoemission from graphene. (The energy levels are for illustration only, not drawn to scale.) (b and c) Spectral dependence of photoexcited electron emission of graphene $/ \mathrm{SiO}_{2} / \mathrm{Si}$ with $\mathrm{Al}_{2} \mathrm{O}_{3}$ and $\mathrm{HfO}_{2}$ top gate, respectively. An expanded view near the threshold is shown in the insets.

Figure 4 is the electron emission from the Fermi level of graphene due to the enhancement of absorption in the graphene layer. For the device with the $\mathrm{HfO}_{2}$ top gate, we observe lower currents produced by electron injection from the graphene and slightly weaker oscillation amplitude as shown in the inset of Figure 3. This is attributed to the lower light transmission of $\mathrm{HfO}_{2}$ than that of $\mathrm{Al}_{2} \mathrm{O}_{3}$ in the entire measured spectrum, which was confirmed by model simulation. For each externally applied field, that is, each bias applied at the silicon substrate, the spectral threshold $\Phi_{\mathrm{e}}$ is extracted by a linear fitting to the cube root of the emission yield within the spectral range less than $1 \mathrm{eV}$ above the threshold (see Figure 4). Two mechanisms are in play in changing the barrier height: (1) an applied electric field ( $F$, defined by the ratio of applied bias to the oxide thickness) causes the barrier to be lowered, which is classically known as the image force lowering; ${ }^{27}$ (2) the Fermi energy of graphene is shifted by the applied electric field. However, in our case with a thick back gate, the latter plays a very insignificant role because the Fermi level of graphene can be easily shown to move at the rate of less than $0.0025 \mathrm{eV}$ per volt. $^{28}$ Thus, the effect of Fermi level shift can be neglected. We determine the barrier height $\left(\Phi_{0}\right)$ at the flat band condition or zero field in the oxide by employing the Schottky coordinates, $\Phi(F)$ vs $F^{1 / 2}$ and plotting the field dependence of the barrier height. ${ }^{14}$ The threshold $\Phi$ is reduced with electric field strength $F$ by the expression:

$$
\Phi(F)=\Phi_{0}-q \sqrt{\frac{q F}{4 \pi \varepsilon_{0} \varepsilon_{i}}}
$$

where $q$ is the carrier charge, $\varepsilon_{0}$ is the dielectric permittivity of vacuum, and $\varepsilon_{i}$ is the effective image-force constant. ${ }^{27}$ Thus, we find $\Phi_{0}$ by linear extrapolation to zero field as shown in Figure 5. The zero-field barrier heights from the Fermi level of

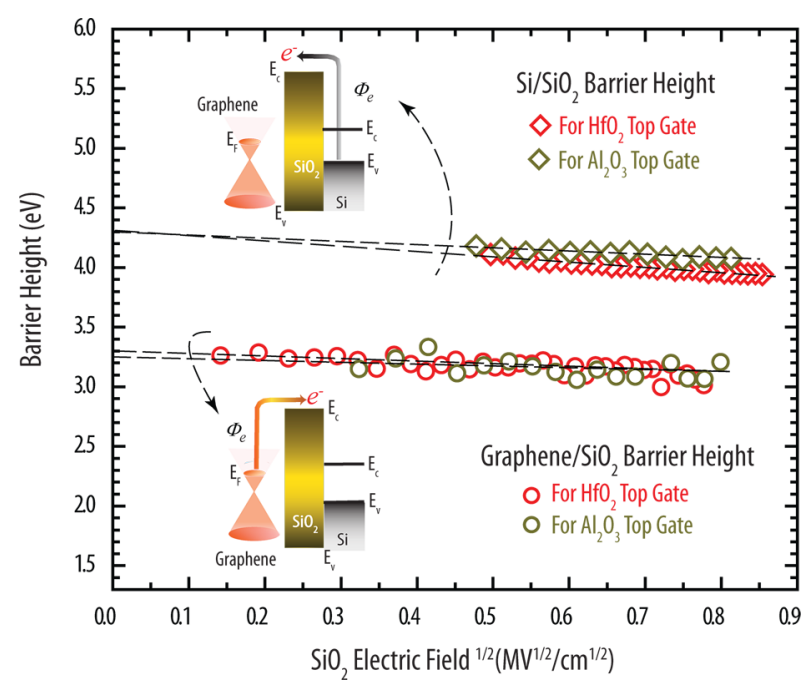

Figure 5. Schottky plot of the field-dependent IPE threshold measured at graphene $/ \mathrm{SiO}_{2}$ interface for test structures with different top gate high- $k$. The measured threshold of $\mathrm{Si} / \mathrm{SiO}_{2}$ is also shown in the plot.

graphene to the conduction band edge of $\mathrm{SiO}_{2}, \Phi_{\mathrm{e}}\left[E_{\mathrm{F}, \text { Graphene }}-\right.$ $\left.\mathrm{CB}_{\mathrm{SiO}_{2}}\right]$, are $3.23 \mathrm{eV} \pm 0.05 \mathrm{eV}$ and $3.30 \mathrm{eV} \pm 0.05 \mathrm{eV}$ for the structure with the $\mathrm{Al}_{2} \mathrm{O}_{3}$ top gate and the $\mathrm{HfO}_{2}$ top gate, respectively.

For completeness, we also extract the barrier height for the $\mathrm{Si} / \mathrm{SiO}_{2}$ interface from the IPE measurements. We observe the barrier from both the valence band (VB) and the conduction band $(\mathrm{CB})$ of $\mathrm{Si}$ to the conduction band of $\mathrm{SiO}_{2}$ for the device with $\mathrm{Al}_{2} \mathrm{O}_{3}$ top gate; $\Phi_{\mathrm{e}}\left[\mathrm{VB}_{\mathrm{Si}}-\mathrm{CB}_{\mathrm{SiO}_{2}}\right]=4.3 \mathrm{eV} \pm 0.05 \mathrm{eV}$ and $\Phi_{\mathrm{e}}\left[\mathrm{CB}_{\mathrm{Si}}-\mathrm{CB}_{\mathrm{SiO}_{2}}\right]=3.23 \mathrm{eV} \pm 0.05 \mathrm{eV}$, both are in close agreement with accepted literature values. ${ }^{21,29,30}$ The presence of electrons being excited from the conduction band of $\mathrm{Si}$, that is, $\Phi_{\mathrm{e}}\left[\mathrm{CB}_{\mathrm{Si}}-\mathrm{CB}_{\mathrm{SiO}_{2}}\right]$, is experimentally uncommon due to the fact that it is only observed in degenerately doped n-type $\mathrm{Si}$. With the $\mathrm{HfO}_{2}$ top gate, the injection from the conduction band of $\mathrm{Si}$ to the conduction band of $\mathrm{SiO}_{2}$ was not discernible due to the relatively lower photocurrents, and therefore, only photoexcited electrons from the valence band of $\mathrm{Si}$ to the conduction band of $\mathrm{SiO}_{2}$ were observed, giving rise to $\Phi_{\mathrm{e}}\left[\mathrm{VB}_{\mathrm{Si}}-\mathrm{CB}_{\mathrm{SiO}_{2}}\right]=4.32 \mathrm{eV}$. 
To generate the complete picture of the graphene $/ \mathrm{SiO}_{2}$ band diagram, in addition to the barrier heights the doping type and the position of Fermi level with respect to the Dirac point in graphene need to be determined. They can be extracted by measuring the currents $\left(I_{\mathrm{DS}}\right)$ between drain $(\mathrm{Cr} / \mathrm{Au})$ and source $(\mathrm{Cr} / \mathrm{Au})$ (see Figure 1$)$ when a varying bias $\left(V_{\mathrm{TG}}\right)$ is applied to the top gate $(\mathrm{Ti} / \mathrm{Pt})$ as depicted in Figure 6. The top

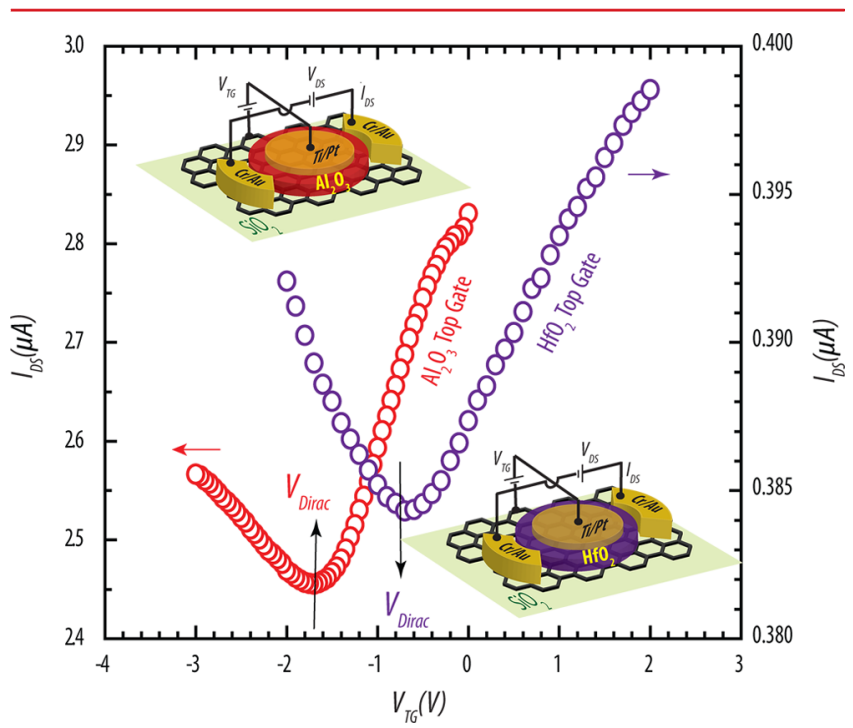

Figure 6. Transfer characteristics of graphene FET with bias at $\mathrm{Al}_{2} \mathrm{O}_{3}$ and $\mathrm{HfO}_{2}$ top gate. The inset structures depict the electrical measurement arrangements performed on two structures with $\mathrm{Al}_{2} \mathrm{O}_{3}$ (top) and $\mathrm{HfO}_{2}$ (bottom).

gate was used here instead of the bottom one because the shift in the Fermi level with respect to the Dirac point is much more sensitive to the applied gate voltage. The gate voltage $V_{\mathrm{TG}}$ creates an electrostatic potential difference $(\varphi)$ between the graphene and the gate electrode and induces an addition of charge carriers in graphene that results in a shift in the graphene Fermi level $\left(E_{\mathrm{f}}\right)$, that is, $V_{\mathrm{TG}}=\left(E_{\mathrm{f}} / q\right)+\varphi$, where $q$ is the elementary charge. Since $\varphi$ is inversely proportional to the geometric gate capacitance and thus proportional to the oxide thickness, it is obvious that $\varphi$ is the predominant term when the oxide thickness is very large and therefore the measured $E_{\mathrm{f}}$ would be less accurate had the bottom gate with the very large oxide been used. ${ }^{28}$ By applying the top gate biases, Figure 6 exhibits Dirac voltages $\left(V_{\text {Dirac }}\right)$ of $-1.7 \mathrm{~V}\left(\mathrm{Al}_{2} \mathrm{O}_{3}\right.$ top gate $)$ and $-0.7 \mathrm{~V}$ ( $\mathrm{HfO}_{2}$ top gate), respectively, and the Fermi level shift $\left(E_{\mathrm{f}}\right)$ with respective to the Dirac point can be computed by the expression: ${ }^{30}$

$$
E_{\mathrm{f}}=\hbar v_{\mathrm{F}} \sqrt{\pi \alpha\left(V_{\mathrm{G}}-V_{\text {Dirac }}\right)}
$$

where $\alpha=\left(C_{\mathrm{ox}} / q\right), v_{\mathrm{F}}$ is the known graphene Fermi velocity, and $C_{\mathrm{ox}}$ is the geometric capacitance of the top gate oxide (calculated from the known dielectric constant of the top gate dielectric and its thickness). To be accurate, a stack fabricated with the same process was used to determine the dielectric constant by using a dual gate measurement. ${ }^{31}$ As a result, Fermi level shifts $\left(E_{\mathrm{f}}\right)$ are found to be $0.33 \mathrm{eV}\left(\mathrm{Al}_{2} \mathrm{O}_{3}\right.$ top gate $)$ and $0.30 \mathrm{eV}\left(\mathrm{HfO}_{2}\right.$ top gate) above the Dirac point in graphene when there is no applied gate bias or $V_{\mathrm{G}}=0$.

Having measured the position of the graphene Fermi level relative to both the bottom of the $\mathrm{SiO}_{2}$ conduction band as well as its Dirac point, we are able to construct the complete electronic band diagram as depicted in Figure 7. Summing the Fermi level position of graphene and the electron barrier height

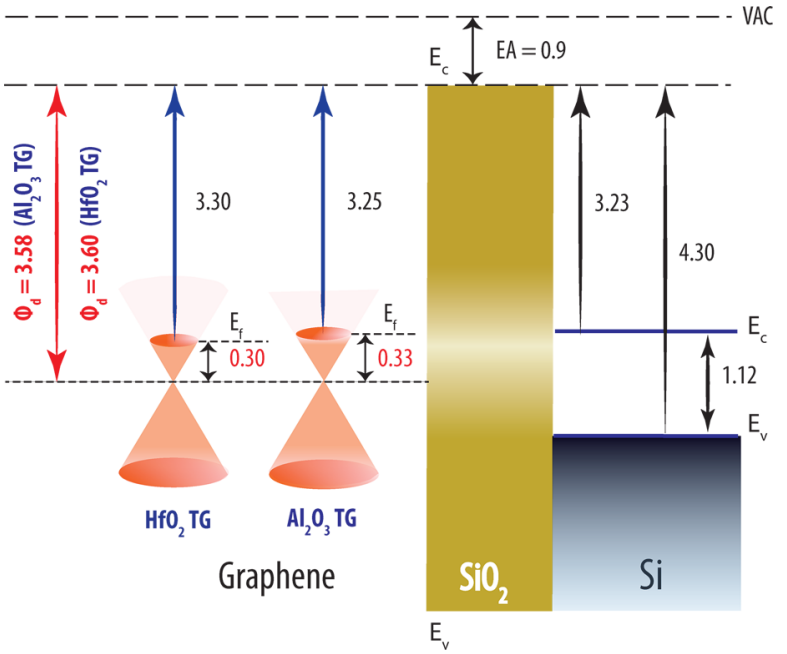

Figure 7. Complete electronic band diagram of graphene $/ \mathrm{SiO}_{2} / \mathrm{Si}$ system; all values are in $\mathrm{eV}$.

$\left(\Phi_{\mathrm{e}}\right)$, the intrinsic barrier height, $\Phi_{\mathrm{i}}=\Phi_{\mathrm{e}}\left[E_{\mathrm{F}, \text { Graphene }}-\mathrm{CB}_{\mathrm{SiO}_{2}}\right]+$ $E_{f}$, precisely yields the graphene Dirac point relative to the conduction band of $\mathrm{SiO}_{2}$. The experimentally derived values are $3.58 \mathrm{eV}$ in the case of the $\mathrm{Al}_{2} \mathrm{O}_{3}$ top gate and $3.60 \mathrm{eV}$ in the case of the $\mathrm{HfO}_{2}$ top gate. With the well-established electron affinity $^{32}$ (EA) of $\mathrm{SiO}_{2}$ of $0.9 \mathrm{eV}$ and the measured intrinsic barrier height, we find the intrinsic work function of undoped graphene (the Dirac point) to be $4.48 \mathrm{eV} \pm 0.05 \mathrm{eV}\left(\mathrm{Al}_{2} \mathrm{O}_{3}\right.$ top gate) and $4.50 \mathrm{eV} \pm 0.05 \mathrm{eV}\left(\mathrm{HfO}_{2}\right.$ top gate), which are in very close agreement with previous reports. ${ }^{9-11,13}$ Importantly, our findings show that the barrier height at the graphene $/ \mathrm{SiO}_{2}$ interface is insensitive within the precision of our measurements to the presence of the top gate. This is consistent with recent atomic force microscopy studies reporting that graphene is attached to oxides only by the relatively weak van der Waals interaction, ${ }^{33}$ and therefore the effects of the top gate dialectic would be minimal to the intrinsic work function of graphene.

In conclusion, we have used the internal photoemission method on a uniquely designed optical-cavity graphene $/ \mathrm{SiO}_{2} /$ $\mathrm{Si}$ test structure to directly measure the barrier height from the Fermi level of large-area CVD single layer graphene to the conduction band edge of $\mathrm{SiO}_{2}$. A complementary electrical measurement was made on the same test structure from which we obtain the Fermi level position with respect to the Dirac point of graphene. By using the knowledge gained from these correlated measurements and the known functional dependence of the density of states near the Dirac point, we determine the intrinsic barrier height of graphene (Dirac point of graphene to the conduction band edge of $\left.\mathrm{SiO}_{2}\right)$, as well as the intrinsic work function of graphene. Such fundamental insights into the band alignment at the graphene-insulator interface are a necessary and important advancement toward the rational design, fabrication, and implementation of graphene-based electronic and optical devices.

\section{AUTHOR INFORMATION}

\section{Corresponding Author}

*E-mail: xu83@purdue.edu (K.X.); nhan.nguyen@nist.gov (N.V.N.). 


\section{Author Contributions}

${ }^{\perp}$ These authors contributed equally.

\section{Notes}

The authors declare no competing financial interest.

\section{ACKNOWLEDGMENTS}

The authors (C.Z. \& K.W.) acknowledge support from the Focus Center Research Program (FCRP)-Functional Engineered Nano Architectonics (FENA).

\section{REFERENCES}

(1) Novoselov, K. S.; Geim, A. K.; Morozov, S. V.; Jiang, D.; Zhang, Y.; Dubonos, S. V.; Grigorieva, I. V.; Firsov, A. A. Science 2004, 306, 666-669.

(2) Cooper, D. R.; D’Anjou, B.; Ghattamaneni, N.; Harack, B.; Hilke, M.; Horth, A.; Majlis, N.; Massicotte, M.; Vandsburger, L.; Whiteway, E.; Yu, V. ISRN Condens. Matter Phys. 2012, 2012, 1-56.

(3) Castro Neto, A. H.; Guinea, F.; Peres, N. M. R.; Novoselov, K. S.; Geim, A. K. Rev. Mod. Phys. 2009, 81, 109-162.

(4) Das Sarma, S.; Adam, S.; Hwang, E. H.; Rossi, E. Rev. Mod. Phys. 2011, 83, 407-470.

(5) Kim, K. S.; Zhao, Y.; Jang, H.; Lee, S. Y.; Kim, J. M.; Kim, K. S.; Ahn, J.-H.; Kim, P.; Choi, J.-Y.; Hong, B. H. Nature 2009, 457, 706710.

(6) Bae, S.; Kim, H.; Lee, Y.; Xu, X.; Park, J.-S.; Zheng, Y.; Balakrishnan, J.; Lei, T.; Kim, H. R.; Song, Y. I.; Kim, Y.-J.; Kim, K. S.; Özyilmaz, B.; Ahn, J.-H.; Hong, B. H.; Iijima, S. Nat. Nanotechnol. 2010, 5, 574-578.

(7) Kedzierski, J.; Hsu, P.-L.; Reina, A.; Kong, J.; Healey, P.; Wyatt, P.; Keast, C. IEEE Electron. Device Lett. 2009, 30, 745-747.

(8) Moriyama, S.; Morita, Y.; Watanabe, E.; Tsuya, D.; Uji, S.; Shimizu, M.; Ishibashi, K. Sci. Technol. Adv. Mater. 2010, 11, 054601.

(9) Ziegler, D.; Gava, P.; Guettinger, J.; Molitor, F.; Wirtz, L.; Lazzeri, M.; Saitta, A. M.; Stemmer, A.; Mauri, F.; Stampfer, C. Phys. Rev. B 2011, 83, 235434.

(10) Yu, Y.-J.; Zhao, Y.; Ryu, S.; Brus, L. E.; Kim, K. S.; Kim, P. Nano Lett. 2009, 9, 3430-3434.

(11) Filleter, T.; Emtsev, K. V.; Seyller, T.; Bennewitz, R. Appl. Phys. Lett. 2008, 93, 133117-133117-3.

(12) Khomyakov, P. A.; Giovannetti, G.; Rusu, P. C.; Brocks, G.; Van den Brink, J.; Kelly, P. J. Phys. Rev. B 2009, 79, 195425.

(13) Song, S. M.; Park, J. K.; Sul, O. J.; Cho, B. J. Nano Lett. 2012, 12, 3887-3892.

(14) Afanas'ev, V. V. Internal Photoemission Spectroscopy: Principles and Applications; Elsevier: Amsterdam, 2008.

(15) Nguyen, N. V.; Xu, M.; Kirillov, O. A.; Ye, P. D.; Wang, C.; Cheung, K.; Suehle, J. S. Appl. Phys. Lett. 2010, 96, 052107-052107-3.

(16) Zhang, Q.; Zhou, G.; Xing, H. G.; Seabaugh, A. C.; Xu, K.; Sio, H.; Kirillov, O. A.; Richter, C. A.; Nguyen, N. V. Appl. Phys. Lett. 2012, 100, 102104-102104-4.

(17) Yan, R.; Zhang, Q.; Li, W.; Calizo, I.; Shen, T.; Richter, C. A.; Hight-Walker, A. R.; Liang, X.; Seabaugh, A.; Jena, D.; Grace Xing, H.; Gundlach, D. J.; Nguyen, N. V. Appl. Phys. Lett. 2012, 101, 022105022105-4.

(18) Nair, R. R.; Blake, P.; Grigorenko, A. N.; Novoselov, K. S.; Booth, T. J.; Stauber, T.; Peres, N. M. R.; Geim, A. K. Science 2008, $320,1308-1308$.

(19) Hsu, A.; Wang, H.; Kim, K. K.; Kong, J.; Palacios, T. IEEE Electron. Device Lett. 2011, 32, 1008-1010.

(20) Nguyen, N. V.; Kirillov, O. A.; Suehle, J. S. Thin Solid Films 2011, 519, 2811-2816.

(21) Powell, R. J. J. Appl. Phys. 1970, 41, 2424-2432.

(22) Fowler, R. H. Phys. Rev. 1931, 38, 45-56.

(23) Ando, T. NPG Asia Mater. 2009, 1, 17-21.

(24) Weber, J. W.; Calado, V. E.; Van de Sanden, M. C. M. Appl. Phys. Lett. 2010, 97, 091904-091904-3.

(25) In Handbook of Optical Constants of Solids; Academic Press: Burlington, 1997; pp 5-114.
(26) Heavens, O. S. Optical Properties of Thin Solid Films; Courier Dover Publications: North Chelmsford, MA, 2011.

(27) Sze, S. M. Semiconductor Devices: Physics and Technology, 2nd ed.; Wiley: New York, 2001

(28) Das, A.; Pisana, S.; Chakraborty, B.; Piscanec, S.; Saha, S. K.; Waghmare, U. V.; Novoselov, K. S.; Krishnamurthy, H. R.; Geim, A. K.; Ferrari, A. C.; Sood, A. K. Nat. Nanotechnol. 2008, 3, 210-215.

(29) Goodman, A. Phys. Rev. 1966, 144, 588-593.

(30) Mead, C. A. Appl. Phys. Lett. 1965, 6, 103.

(31) Fallahazad, B.; Lee, K.; Lian, G.; Kim, S.; Corbet, C. M.; Ferrer, D. A.; Colombo, L.; Tutuc, E. Appl. Phys. Lett. 2012, 100, 093112093112-4.

(32) Robertson, J. Eur. Phys. J. Appl. Phys. 2004, 28, 265-291.

(33) Song, S. M.; Cho, B. J. Nanotechnology 2010, 21, 335706.

\section{NOTE ADDED AFTER ASAP PUBLICATION}

This article was published ASAP on December 20, 2012. Due to a production error, the graphs for Figures 5 and 6 were interchanged. The correct version was published on December $21,2012$. 